\title{
介电晶体光波导的制备及其应用
}

\author{
贾曰辰, 王士香, 谭杨, 王磊, 陈峰* \\ 山东大学物理学院, 晶体材料国家重点实验室, 济南 250100 \\ * 联系人, E-mail: drfchen@sdu.edu.cn
}

2020-12-30 收稿, 2021-02-01 修回, 2021-02-02 接受, 2021-02-03 网络版发表

国家重点研发计划(2019YFA0705000)、国家自然科学基金(11535008, 61775120，12074223)、山东省泰山学者青年专家计划(tsqn201909041)和 山东大学齐鲁青年学者项目资助

\begin{abstract}
摘要介电光学晶体种类繁多, 具有丰富的功能属性, 在科学研究、生产生活的各个领域有着广泛的应用. 光波导 是基本的光学微结构之一。基于介电光学晶体的光波导结构是组成集成光子学器件的重要元件. 利用飞秒激光直 写或者载能离子束辐照技术, 可以有效调控晶体材料局部区域的折射率分布, 形成低损耗的光波导结构. 本文将介 绍介电晶体光波导(包括单晶薄膜)的制备方法及相关的波导性能, 综述介电晶体光波导在激光产生、非线性光学 频率转换、信号调制以及量子信息中的应用, 并对这一领域的未来研究进行展望.
\end{abstract}

关键词介电晶体, 光波导, 飞秒激光微加工, 离子注入, 铌酸锂单晶薄膜

光波导是集成光子学、光电子学的基本元件, 在 现代光通讯、光信息存储、量子计算、非线性光学以 及微型激光源制造等领域中具有重要的应用 ${ }^{[1 \sim 4]}$. 由于 波导结构的尺度小(其截面一般为微米尺度), 光波导可 以把光的能量限制于体积微小的通道内, 达到很高的 光强密度, 使得基于波导结构的激光产生阈值更低, 非 线性频率转换效率更高 ${ }^{[1 \sim 6]}$. 同时, 在波导结构中, 光的 传输可以被有效地、无衍射地引导，构建出高度集成 化的光波导器件和波导网络. 波导结构的基质一般包 括半导体晶体(如硅等)、光学玻璃(如熔融石英、磷酸 盐、硅酸盐玻璃等)、介电光学晶体(如 $\mathrm{LiNbO}_{3} 、 \mathrm{Nd}$ : $\mathrm{YAG} 、 \mathrm{KTiOPO}_{4}(\mathrm{KTP})$ 等人工功能晶体) 等 $^{[1,2]}$. 介电光 学晶体是重要的光学介质, 不同功能、不同结构的光 学晶体在多个领域发挥着不可替代的作用 ${ }^{[72]}$. 例如, 与激光玻璃相比, 以激光晶体为增益介质的固体激光 核心增益组件体积更小, 发射光谱线宽更窄(单色性 好); 非线性光学晶体可以实现激光频率转换, 这在玻 璃、硅基材料中是很难实现的. 此外, 许多介电光学晶
体还具备电光、声光、压电等性能，在工业生产、日 常生活、科学研究以及国防建设等多个领域发挥着重 要的作用. 基于介电光学晶体的光波导结构结合了晶 体材料与波导微结构各自的优势，是集成光子学的重 要平台, 在诸多领域具有广阔的应用前景 ${ }^{[1,2]}$.

载能离子束注人/辐照技术是制备晶体光波导的重 要方法 ${ }^{[3,10,11]}$. 由于晶体结构的复杂性以及化学、光 学、机械属性的特殊性, 目前已发展的许多波导制备 方法大多对一种或者几种特定的晶体有效. 例如, 金属 离子热扩散法、质子交换法等化学方法可以应用于 $\mathrm{LiNbO}_{3}$ 晶体, 却对化学性质稳定的大多数晶体无效. 作 为“物理”方法，离子注人/辐照技术基于注人离子与晶 体晶格的相互作用产生局域化的晶格畸变或缺陷，通 过晶格应力场或者体密度的变化来改变晶体特定区域 的折射率, 并在适当条件下形成光波导结构. 这种方法 不依赖于晶体的化学性质, 理论上可以在任何温度下 进行, 大大拓展了离子束技术对材料的适用范围 ${ }^{[3,10,11]}$. 离子束注人/辐照技术既可以制备折射率改变均匀、大

引用格式: 贾曰辰, 王士香, 谭杨, 等. 介电晶体光波导的制备及其应用. 科学通报, 2021, 66: 1968-1982 Jia Y C, Wang S X, Tan Y, et al. Fabrication and applications of dielectric optical crystalline waveguides (in Chinese). Chin Sci Bull, 2021, 66: 1968-1982, doi: 10.1360/TB-2020-1686 
面积的平面波导 ${ }^{[3,10]}$ ，也可以通过“切片”的方式制备亚 微米厚度的单晶薄膜 ${ }^{[10,12]}$, 在波导的模式调控上有很 大的灵活度．离子束技术用于制备光波导可以大致分 为离子注人 (又包括轻、重离子注人)、快重离子辐 照、聚焦离子束写人(一般使用质子或氦离子)、离子 束切片以及离子束增强刻蚀等 ${ }^{[3]}$. 它们对晶体的作用机 理各不相同, 作用效果也很不一样. 根据晶体的种类和 属性，利用适当的离子束方法可以制备具有不同功能 的晶体光波导.

飞秒激光直写技术可以用来制备多功能微纳光子 学结构 ${ }^{[4,6,13 \sim 18]}$. 飞秒激光束的光功率密度高, 作用在光 学材料中微米、亚微米尺度的区域, 可以诱导双光子 及多光子吸收, 实现精细的微纳加工. 通过这种激光直 写方法制备的光波导结构性能优良, 模式可以调控, 传 输损耗低. 最为重要的是它强大的三维微纳加工能力, 已被广泛用于制备基于透明材料的三维光波导结 构 ${ }^{[4,6,13]}$. 然而, 这种制备三维波导的能力严重依赖激光 辐照(直写)区域的折射率变化. 玻璃是非晶的各向同性 介质, 对于这类光学材料, 飞秒激光一般会在辐照区诱 导折射率增大 (即 $\Delta n>0$, 通常称为第一类折射率改性, 直写区即为波导结构 $)^{[4,6]}$, 再通过三维扫描样品，就可 直接形成三维波导通道, 实现三维结构的光波导器件. 这种三维波导不仅在传统的光通讯领域有重要应用, 最近还被用来构建量子芯片 ${ }^{[19]}$ 以及实现光的拓扑绝缘 体 ${ }^{[20]}$. 对于非线性光学晶体这样的各向异性材料, 飞秒 激光在直接作用区大多会形成折射率减小（即 $\Delta n<0$, 通 常称为第二类折射率改性)的刻痕( track), 不会直接诱 导产生波导; 在刻痕周围区域, 飞秒激光辐照形成的应 力场引起折射率增大, 形成波导核心 ${ }^{[4,21]}$. 然而, 波导结 构的属性由于晶体材料结构本身的原因具有相当的复 杂性. 一方面, 非线性光学晶体材料都具有各向异性, 而且晶格结构多种多样; 另一方面, 即使是同一种晶 体, 不同晶向对飞秒激光的响应也可能不一样.一般来 说，飞秒激光写人晶体波导多采用“双线”结构(两条飞 秒激光诱导的刻痕之间区域由于应力作用形成波导核 心), 或者采用“凹陷包层”或“包层”结构(也称第三类波 导结构, 由一些紧密排列的平行刻痕 $(\Delta n<0)$ 包围形成 折射率相对增强的波导核心). 由于晶体结构的复杂性, 对于 $\Delta n<0$ 的非线性光学晶体来说，用常用的飞秒激光 制备方案很难实现三维波导器件 ${ }^{[4,6]}$. 对少数晶体(如 $\mathrm{LiNbO}_{3}$ ) 来说, 飞秒激光可以直接诱导辐照区在某一特 定偏振方向上形成波导(异常光折射率增大, 即 $\Delta n_{\mathrm{e}}>0$ ).

\section{1 飞秒激光微加工技术制备的光波导}

\section{1 飞秒激光与物质相互作用基本原理}

与使用长脉冲激光(如纳秒量级)以及其他技术手 段进行高精度微加工相比，聚焦的飞秒激光脉冲可以 在尺度很小的区域对材料进行改性及修饰，并且对周 围区域的影响极小. 其主要原因在于, 飞秒激光脉冲与 物质相互作用的时间尺度极短，电子和离子的热耦合 在激光脉冲持续时间尺度内可忽略不计，在加工过程 中也几乎没有热效应(特别是在低重复频率激光作用 下 $)^{[4,13]}$. 基于这个独特的优点, 飞秒激光微加工技术在 激光精密加工领域得到了广泛的关注和应用，尤其是 在透明介质的微纳加工方面展现出了强有力的功能性 和灵活度.

在飞秒激光对应的波长下具有光学透明的性质是 在特定光学晶体中进行有效飞秒激光微纳加工的前提 条件. 常见的飞秒激光器的输出波长为 $800 \mathrm{~nm}$ (Ti:Sapphire 飞秒激光器)以及1.03 1.05 $\mu \mathrm{m}$ ( $\mathrm{Yb}$ 掺杂光纤飞秒 激光器). 由于具有较大的带隙, 大多数光学晶体材料 在这两个波长都具有良好的透明度，因此非常适 合利用飞秒激光进行微纳加工. 当飞秒激光脉冲聚 焦 在透明介质晶体材料表面时，电子将通过强场电 离过 程(多光子电离或隧穿电离)从其母原子上脱离 ${ }^{[4,13]}$. 为 了获得大量的自由电子, 此电离过程通常需要 $10^{12} \sim 10^{13} \mathrm{~W} / \mathrm{cm}^{2}$ 的激光强度. 这些产生的自由电子将 继续与激光脉冲相互作用, 并通过逆轫致辐射吸收(inverse bremsstrahlung absorption)过程吸收更多的能量, 与其他将要释放的束缚电子发生碰撞(雪崩电离过程, avalanche ionization). 通过一系列的非线性吸收和电离 过程，受到飞秒激光脉冲辐照的材料区域将会主要由 高温致密的电子等离子体(electron plasma)以及离子晶 格所组成 ${ }^{[4,13]}$. 激光与物质相互作用过程之后, 能量的 转移过程非常复杂, 并且在很大程度上取决于被加工 材料和激光的各项参数，其物理图像和模型也尚未完 善清晰 ${ }^{[4,13]}$. 通常, 如果激光的强度足够高(一般达到几 个 $\mathrm{J} / \mathrm{cm}^{2}$ ), 并产生了较高的等离子体密度, 则激光将会 由于库仑爆炸效应(Coulomb explosion effect)在材料的 表面产生火山口形状的烧蚀微坑，其边缘区域由于不 受热效应的影响将会非常光滑 ${ }^{[4,13]}$. 在这个过程中, 如 果样品和激光聚焦的相对位置保持不变，多次飞秒激 光脉冲的辐照会在样品表面形成更深的烧蚀微坑; 而 在样品以一定速度移动的情况下，则会形成沿着移动 
方向的烧蚀凹槽. 这些技术手段都已经成功应用于光 学晶体表面微结构制备的精密加工, 制备了多种多样 的光子学器件 ${ }^{[4,13]}$. 此外, 利用超快激光烧蚀可以通过 两种途径在光学晶体中制备光学波导结构: 一种是利 用激光烧蚀在附近未加工材料区域产生的冲击波 (shock wave)导致局域化的折射率增加, 并简单地沿晶 体表面产生消融凹槽, 可以用于光波导结构的制 备 ${ }^{[4,13]}$; 另一种方案是在利用其他技术(如离子注人技 术)制备的平面光波导的基础上, 通过对样品表面的激 光烧蚀, 形成两个平行的烧蚀凹槽来提供横向的光学 限制, 从而制备脊型光波导结构(也称为第四类波导结 构 $)^{[4,13]}$. 当飞秒激光脉冲聚焦在介电晶体材料内部的 某个特定深度时, 高强度的激光与物质相互作用的过 程将变得更加复杂 ${ }^{[4,13]}$. 脉冲在晶体内部传输的过程中 将经历非线性过程, 使得其光场强度分布与在低强度 下的分布完全不同. 影响脉冲传输的机制较为复杂, 主 要与材料的三阶非线性极化率以及光学克尔效应有关. 对于较大的脉冲激光功率, 飞秒激光光束将会经历自 聚焦效应(self-focusing), 进一步提升局部的同轴光强 度. 最终, 在光脉冲聚焦位置附近的局部区域将会发生 强场电离效应, 产生的高温、高密度的等离子体会倾 向于使光束散焦(defocusing), 并同时从脉冲中吸收能 量. 其中, 光学自聚焦和等离子体生成(散焦)的相反效 果有时会产生动态光通道, 在该通道中, 光束以非常小 的尺寸集中传播, 聚焦距离为几个瑞利长度(Rayleigh lengths), 该过程被称为丝化(filamentation) ${ }^{[4,13]}$. 基于当 前的研究结果, 在飞秒激光聚焦位置产生的等离子体 被认为是产生局域化的材料改性的主要原因. 然而, 自 由电子等离子体将其能量转移到局域化的晶格之后引 发的损伤机制尚不清晰, 其物理机制也尚未被完全理 解 ${ }^{[4,13]}$.

\section{2 飞秒激光直写光波导的基本制备手段}

选取合适的飞秒激光光源对于制备高性能的介电 晶体光波导非常重要, 尤其是针对基于不同类型折射 率改性机制的波导结构. 通常来说, 由于第一类折射率 改性所需要的飞秒激光能量较低, 因此可以利用峰值 功率较低的飞秒激光振荡器来实现. 与此相对, 基于第 二类折射率改性机制的飞秒激光直写则需要峰值功率 较高的飞秒激光器来进行. 除此之外, 在飞秒激光直写 过程中, 激光的扫描速率和重复频率对材料改性机制 和制备波导的性能有很大的影响. 例如, 当所需要的激
光峰值功率较低时, 可以采用较快的飞秒激光扫描速 度(或样品移动速度) 以及较高的飞秒激光重复频率. 反 之, 当需要较高的激光峰值功率时, 可以降低激光的扫 描速度(或样品的移动速度)或者降低所使用飞秒激光 的重复频率. 在飞秒激光直写过程中, 通常将待加工样 品放置在可进行精密位置和俯仰调节的三维机械调节 台上, 利用透镜或者物镜将人射的飞秒激光聚焦在代 加工样品的特定位置, 进行局域化的激光改性. 在加工 过程中, 样品的移动(即飞秒激光的扫描)通常通过改变 三维调节台的位置来实现.

比较常用的飞秒激光直写光波导结构的形式主要 包括纵向写人和横向写人两种(图 1$)^{[4,13]}$. 对于纵向写人 方式, 脉冲激光人射方向与扫描样品的方向平行, 其聚 焦位置在加工过程中慢慢远离或靠近样品. 人射激光束 由于具有高对称性的高斯强度分布, 因而能够制备对称 性较好的圆柱形波导结构. 然而, 纵向写人制备的光波 导长度受聚焦元件(物镜或透镜)的工作距离(working distance)的限制. 在加工过程中, 通常使用的聚焦物镜数 值孔径(numerical aperture, NA)为 0.4 , 其工作距离约为 $5 \mathrm{~mm}$. 对于横向写人, 激光扫描样品的方向垂直于人射 激光方向, 制备的光波导位于样品表面以下几毫米深 度, 其长度和结构不再受聚焦元件工作距离的限制, 非 常适合制备灵活多样的集成光子学器件. 另外, 研究人 员在Nd:YAG陶瓷中制备基于第二类折射率改性的光波 导结构时, 采用了新颖的螺旋状扫描方式, 在不影响波 导性能的基础上大大降低了制备周期 ${ }^{[22]}$.

\section{3 飞秒激光直写光波导器件的应用}

利用飞秒激光微加工制备的光波导结构在解决光 子学系统的诸多技术问题时都具有独特的优势. 本节 将对其中基于波导的电光调制器、非线性频率转换器 和激光器等应用进行总结概述.

\subsection{1 电光调制器}

电光调制器是通过电压或电场的变化对信号光的 相位、强度以及偏振等参数进行调制的器件, 在高 速、短距离光通信领域中具有重要作用, 是重要的集 成光子学器件之一. 特别是基于光波导结构的小型、 可集成电光调制器件, 在当今日趋小型化、集成化的 光子学系统中发挥了不可替代的作用. 其中, 利用飞秒 激光微加工技术, 可以在铌酸锂晶体中制备基于马赫 曾德尔干涉仪(Mach-Zehnder interferometer, MZI)结构 的波导电光调制器, 其波导制备主要是基于第二类折 


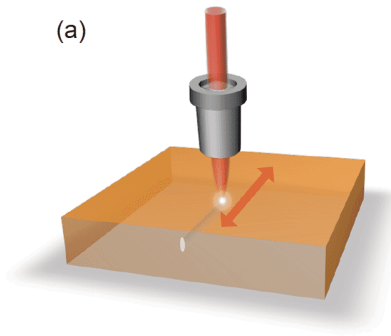

(b)

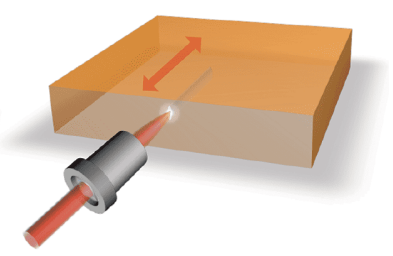

图 1 (网络版彩色)飞秒激光直写介电晶体波导结构的横向(a)和纵 向(b)写人方式示意图

Figure 1 (Color online) Longitudinal (a) and transverse (b) writing geometries for waveguide fabrication in dielectric crystals by femtosecond laser direct writing

射率改性机制 ${ }^{[23 \sim 27]}$. Liao等人 ${ }^{[25]}$ 在飞秒激光直写 $x$ 切的 铌酸锂中制备了波导MZI结构, 其结构由双线型光波导 以及嵌人式的微电极组成, MZI调制器的电光重叠积分 为 0.95 . 随后, Ringleb 等人 ${ }^{[24]}$ 实现了基于飞秒激光直写 铌酸锂光波导的非线性光学频率转换器和电光调制器 (MZI结构)的单片集成. 利用激光烧蚀在样品表面溅射 的金膜制备的电极以及施加合适的电压，该电光调制 器件的电光重叠积分为 0.16 , 半波电压为 $23 \mathrm{~V}$. 此外, Horn等人 ${ }^{[26]}$ 报道了利用低重复频率的飞秒激光制备波 导布拉格光栅结构, 并在通讯波段实现了可调的电光 调制功能. 当施加的电场从 $-22 \mathrm{~V} / \mu \mathrm{m}$ 升高到 $+22 \mathrm{~V} / \mu \mathrm{m}$ 时，电光效应引发的折射率变化使得信号光波长改变 了 $625 \mathrm{~nm}$. 随后，Kroesen等人 ${ }^{[27]}$ 制备了集成在飞秒激 光直写 $\mathrm{LiNbO}_{3}$ 包层波导中的布拉格光栅结构(利用飞 秒激光多次扫描制备, 如图2所示), 该混合结构在通讯 波段具有低损耗、对称的导波模式以及窄带宽的反射 性能, 其电光系数 $\left(r_{13}=7.59 \mathrm{pm} \mathrm{V}^{-1}\right.$ 和 $\left.r_{33}=23.21 \mathrm{pm} \mathrm{V}^{-1}\right)$ 与体材料中相差无几.

\subsection{2 非线性光学频率转换器}

非线性光学晶体能够实现频率转换效应，是获得 多波长和可调谐激光光源最有效的技术手段之一. 由 于光波导紧凑的结构以及对光的限制能力, 波导结构 中具有很高的光密度, 可以制备转换效率优于体材料 的波导频率转换器. 对于飞秒激光直写波导频率转换 器, 迄今为止大部分都集中在二次谐波产生(second harmonic generation)/倍频(frequency doubling)这一非 线性光学频率转换效应上, 并已经成功在 $400 \sim 790 \mathrm{~nm}$ 的波长范围内实现了倍频光输出 ${ }^{[4]}$, 所利用的基质材 料包括 $\mathrm{LiNbO}_{3}{ }^{[28 \sim 34]} 、 \mathrm{KTP}^{[35 \sim 38]} 、 \mathrm{BiB}_{3} \mathrm{O}_{6}(\mathrm{BIBO})^{[39 \sim 41]}$ 等几种非线性光学晶体 ${ }^{[42-46]}$. 这里需要特别指出的是, 由于飞秒激光直写“单线型”和“双线型”光波导一般仅
仅能够支持一个偏振方向的导模，因此只能够实现基 于准相位匹配的倍频效应. 而包层光波导可以在各个 偏振方向下实现有效的导波传输, 相较而言, 在双折射 相位匹配和二次谐波产生方面具有一定的优势.

在波导非线性光学频率转换效应产生的实验装置 中, 其激励光源(脉冲光源或连续光源)的选择取决于相 位匹配条件所需的光源波长以及波导的质量. 一般而 言, 由于峰值功率较高, 脉冲光源激励的非线性光学频 率转换的效率更高, 可以在一定程度上弥补由波导损 耗导致的负面效应. 下面将对两种不同光源激励下的 波导倍频效应进行简单总结. 在脉冲基频光激励的情 况下, 倍频绿光输出已经在飞秒激光直写 $\mathrm{LiNbO}_{3}$ 、 KTP、PPLN(periodically poled $\mathrm{LiNbO}_{3}$ )、PPKTP(periodically poled $\mathrm{KTiOPO}_{4}$ )、PPLT(periodically poled Li$\mathrm{TaO}_{3}$ )、BIBO和 $\mathrm{Nd}: \mathrm{GdCOB}$ 等几种晶体波导中得以实 现 ${ }^{[28,31,35,36,38,40 \sim 44]}$. 其中, $\mathrm{LiNbO}_{3}$ 双线型光波导具有最高 的转换效率, 实验中测得的高达 $49 \%^{[28]}$; KTP包层光波 导具有最高的峰值功率输出, 达到了 $427 \mathrm{~W}^{[35]}$. 对于基 于准相位匹配机制的波导倍频绿光，PPLN双线型光波 导的倍频转换效率达到了 $58 \%$, 输出的峰值功率为 $59 \mathrm{~W}^{[31]}$; PPKTP波导的倍频转换效率为 $47.4 \%$, 峰值功 率可达 $252 \mathrm{~W}^{[38]}$; 而近期研究制备的PPLT包层光波导 (其铁电畴反转的周期为扇形分布, 如图3所示)的倍频 转换效率可达 $54.3 \%$, 输出峰值功率为 $282 \mathrm{~W}^{[44]}$. 另外, 波长为近红外光的倍频效应也已经在PPLN双线型光 波导中得以实现 ${ }^{[32,34]}$.

与脉冲基频光激励的波导倍频效应相比，利用连 续基频光激励的报道相对有限，这也是波导在较低功 率下非线性光学频率转换效率较低的原因 ${ }^{[4]}$. 基于准 相位匹配, 研究人员在PPKTP双线型光波导中实现了 波长为 $400 \mathrm{~nm}$ 的倍频光, 其倍频转换效率为 $0.02 \% \mathrm{~W}^{-1} \mathrm{~cm}^{-2[37]}$. PPLN单线型光波导倍频性能在多 次扫描制备的情况下更加优异，倍频转换效率由 $3 \times 10^{-6} \mathrm{~W}^{-1} \mathrm{~cm}^{-2}$ (单次扫描制备的光波导)提升到 $6.5 \%$ $\mathrm{W}^{-1} \mathrm{~cm}^{-2}$ (多次扫描制备的光波导)，提升了 4 个数量 级 ${ }^{[34]}$. 此外, 研究人员在PPKTP单线型光波导中实现了 倍频蓝光的产生, 转换效率为 $0.18 \% \mathrm{~W}^{-1} \mathrm{~cm}^{-2[37]}$. 近期, Wang 等人 ${ }^{[43]}$ 和 $\mathrm{Li}$ 等人 ${ }^{[44]}$ 在基于PPLT的飞秒激光直写 包层光波导中也成功实现了倍频绿光的产生，通过温 度调节，其倍频转换效率可达 $3.55 \% \mathrm{~W}^{-1} \mathrm{~cm}^{-2[44]}$. 基于 双折射相位匹配机制， Jia等人 ${ }^{[39,40]}$ 在BIBO包层光波导 中实现了比“双线型”光波导高50倍的倍频绿光转换效 


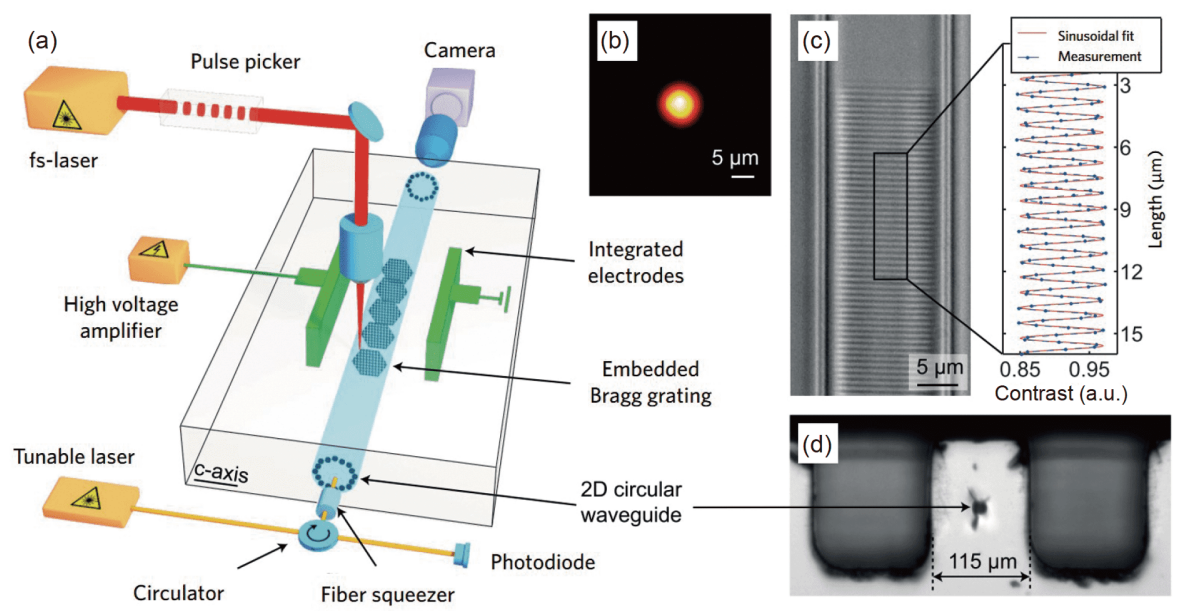

图 2 (网络版彩色)飞秒激光直写制备的嵌人有布拉格光栅的电光波导结构 ${ }^{[27]}$. (a) $\mathrm{LiNbO}_{3}$ 包层波导中布拉格光栅结构直接集成和表征的实验 装置示意图; (b) 圆形端面波导结构的非寻常偏振光模式图; (c) 多次飞秒激光扫描制备的布拉格光栅结构的俯视图; (d) 制备的波导布拉格光栅 和集成的电极端面图(已抛光)

Figure 2 (Color online) Electro-optical tunable waveguide embedded Bragg gratings by direct femtosecond laser writing ${ }^{[27]}$. (a) Schematic of the experimental setup for direct integration and characterization of waveguide embedded Bragg gratings (WBG) in $\mathrm{LiNbO}_{3}$; (b) extraordinary polarized mode of a circular waveguide structure; (c) top view of the multiscan Bragg grating; (d) polished cross section of a WBG with integrated electrodes

(a)

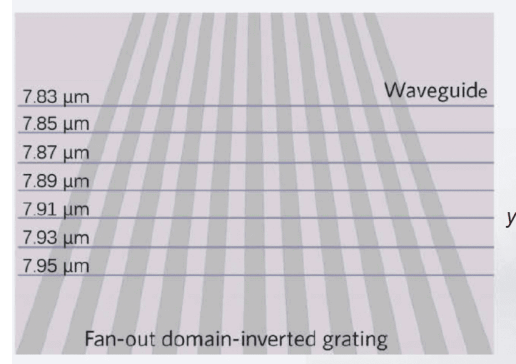

(b)

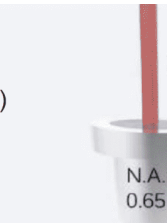

Femtosecond laser
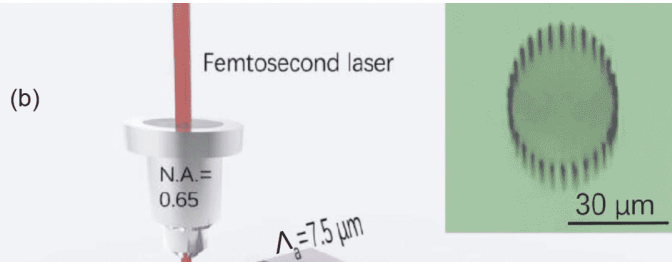
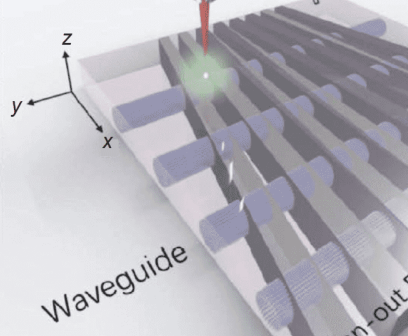

图 3 (网络版彩色) 飞秒激光直写周期极化钽酸锂波导 ${ }^{[44]}$. (a) 周期极化钽酸锂中扇形分布的铁电畴反转示意图; (b) 飞秒激光直写PPLT包层光 波导示意图

Figure 3 (Color online) Femtosecond laser direct writing of fan-out PPLT crystal waveguides ${ }^{[44]}$. (a) The schematic diagram of the fan-out domaininverted grating in PPLT crystal; (b) the schematic plot of femtosecond laser direct writing of cladding waveguides

率(转换效率由 $0.015 \% \mathrm{~W}^{-1}$ 提升到了 $0.75 \% \mathrm{~W}^{-1}$ ), 充分 体现了包层光波导在非线性频率转换效应中的巨大优 势. 另外，Jia等人 ${ }^{[41]}$ 还在BIBO包层光波导中实现了倍 频紫光的产生，其倍频转换效率为 $0.32 \%$ (相当于 $0.98 \% \mathrm{~W}^{-1}$ ).

\section{3 .3 波导激光}

波导激光是紧凑型的激光光源器件. 由于光在波 导结构中能够达到很高的光密度, 因此与体材料激光
相比，基于波导结构的激光器件具有较低的激光國 值、较高的激光效率以及更加灵活、紧凑的几何结构. 迄今为止，基于第二类折射率改性的“双线型”和包层 光波导的波导激光已经在多种晶体/陶瓷中得以实现， 而基于第一类折射率改性的“单线型”光波导结构的波 导激光还很有限 ${ }^{[4]}$. 根据激光增益介质中掺杂的离子种 类, 其激光的输出波长会有所不同. 迄今为止，已经报 道的飞秒激光直写晶体波导激光输出的波长范围覆盖 
了可见光、近红外和中红外波段. 其中, 获得可见光波 段的波导激光输出的一种方式是利用包括 $\mathrm{Nd}$ : $\mathrm{YAB}$ (“双 线型”光波导 $)^{[47]}$ 以及 $\mathrm{Nd}: \mathrm{YCOB}$ (包层光波导) ${ }^{[48]}$ 在内的 自倍频晶体波导, 已经实现的最大输出功率为 $0.1 \mathrm{~mW}$; 另外一种是利用直接光泵浦镨 $(\mathrm{Pr})$ 掺杂晶体波导的方 式, 例如, 利用合适的激光二极管泵浦 $\mathrm{Pr}: \mathrm{SrAl}_{12} \mathrm{O}_{19}{ }^{[49]}$ 和

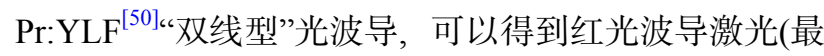
大输出功率为 $28 \mathrm{~mW}$ ). 另外, 研究人员在 Ti:Sapphire“双线型”光波导中获得了可调谐的激光，输出波 长范围为700 870 nm, 最大输出功率为 $143 \mathrm{~mW}$ (波长 为 $800 \mathrm{~nm}$ 时 $)^{[51]}$.

由于其优异的激光性能, $\mathrm{Nd}$ 离子掺杂激光晶体/陶 瓷中的波导激光是该研究方向的重点 ${ }^{[4]}$. Nd:YAG晶体 作为 $\mathrm{Nd}$ 掺杂激光增益介质的典型代表，其波导激光的 研究也受到了研究人员的广泛关注. 迄今为止, 飞秒激 光直写 $\mathrm{Nd}: \mathrm{YAG}$ 波导激光的输出最大功率为 $1.29 \mathrm{~W}$, 利 用的是“双线型”光波导结构 ${ }^{[52]}$. 高的激光斜效率需要 高增益的激光增益介质, 如 $\mathrm{Nd}: \mathrm{YVO}_{4} 、 \mathrm{Nd}: \mathrm{GdVO}_{4}$ 等 $\mathrm{Nd}$ 掺杂饮酸盐激光晶体. 例如, 基于飞秒激光直写 $\mathrm{Nd}$ : $\mathrm{GdVO}_{4}$ “双线型”光波导结构，利用合适的洜浦光源和 波导激光谐振腔，能够获得高达 $70 \%$ 的激光斜效率 ${ }^{[53]}$. 此外，包层光波导结构也已经在其他类型 $\mathrm{Nd}$ 掺杂激光 晶体和陶瓷中实现 ${ }^{[4-6]}$, 由于其偏振无关的优异导波性

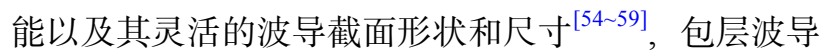
激光的性能以及几何形貌更加灵活, 集成度更高 ${ }^{[60 ~ 64]}$.

除了 $\mathrm{Nd}$ 掺杂的波导激光外，Yb离子掺杂的晶体也 是优良的激光增益介质，如 $\mathrm{Yb}: \mathrm{KGW} 、 \mathrm{Yb}: \mathrm{KYW}$ 和 $\mathrm{Yb}$ : YAG等 ${ }^{[4 ~ 6,65 ~ 68]}$. 其中, 飞秒激光直写 $\mathrm{Yb}$ 掺杂晶体波导 激光的最高输出功率为 $5 \mathrm{~W}^{[66]}$, 最高斜效率为 $75 \%{ }^{[67]}$. 这些高性能的波导激光输出均在Yb:YAG“双线型”光 波导中实现，不同之处在于所利用的泵浦光源(半导体 激光器 ${ }^{[6]}$ 或 Ti:Sapphire激光器 ${ }^{[67]}$ ). 其他类型的飞秒激 光直写波导激光还包括输出波长在中红外波段的(波 长为2 $10 \mu \mathrm{m})$ 波导激光 ${ }^{[69]}$ 、输出波长为双波长的波导

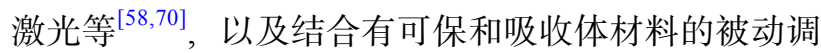
$Q /$ 锁模脉冲波导激光 ${ }^{[4 ~ 6,54,60,71]}$, 这些也是当前以及未 来该领域的几个研究热点.

\section{2 载能离子束技术制备的光波导和单晶薄膜}

\section{1 载能离子束辐照损伤}

在载能离子束辐照介电晶体材料的过程中，高能
的离子束与固体的相互作用是诱导局域化材料损伤的 原因, 这会导致靶材料中的原子核和电子能量损失. 其 中, 核能量和电子能量损失分别与通过弹性碰撞(注人 离子与目标原子核之间)引起的核阻止(nuclear stopping)效应(相对应的阻止本领为 $S_{\mathrm{n}}$ ) 以及通过非弹性碰 撞(注人离子与目标电子之间)引起的电子阻止(electronic stopping) 效应(相对应的阻止本领为 $S_{\mathrm{e}}$ ) 紧密相 关 ${ }^{[3,10]}$. 通常, 描述核能沉积的核阻止功率由 $S_{\mathrm{n}}$ 表示, 而 描述离子诱导的电离效应的电子阻止功率由 $S_{\mathrm{e}}$ 表示. 由 $S_{\mathrm{n}}$ 和 $S_{\mathrm{e}}$ 诱导的晶格损伤在目标材料的物理性质改变以 及在离子辐照区域折射率的变化中起到了至关重要的 作用，因而也是在介电晶体材料中制备有效光波导结 构的关键 $[3,10,11]$

利用载能离子束技术在光学晶体材料中制备包括 光波导、纳米粒子等微纳光子学结构具有较高的灵活 性，通过改变辐照离子的种类、能量、剂量以及离子 束大小，可以实现不同种类、不同尺寸、不同功能的 多功能光子学器件. 一般情况下, 根据辐照离子种类和 能量的不同，可以将载能离子束辐照分为轻离子注 人、重离子注人和快重离子辐照等 ${ }^{[3,10,11]}$.

轻离子注人利用的离子种类通常是质子或氦离子. 为了在介电晶体中制备高性能的光波导结构, 一般采 用的质子注人能量范围约为 $300 \mathrm{keV} \sim 1 \mathrm{MeV}$, 而氦离子 注人典型的能量范围为 $500 \mathrm{keV} 3 \mathrm{MeV}$. 轻离子注人需 要较高的注人剂量 (通常为 $10^{16} \sim 10^{17} \mathrm{~cm}^{-2}$ ), 以形成足够 的晶格损伤来产生波导结构需要的折射率变化 ${ }^{[3,10]}$. 在 轻离子注人的过程中, 其诱导的晶格损伤主要由 $S_{\mathrm{n}}$ 决 定, 大部分发生于注人离子在材料中的射程末端. 而电 子能量损伤在这种情况下的影响很小, 可以忽略. 轻离 子注人的一个特殊应用是制备高质量的晶体薄膜, 这 项技术通常被称为离子切割技术(ion-cut technology) ${ }^{[10]}$. 例如, 为了制备独立式的 $\mathrm{LiNbO}_{3}$ 晶体薄膜(厚 度为几个 $\mu \mathrm{m}$ ), 能量为 $2 \sim 3 \mathrm{MeV}$ 的氦离子注人已经被证 明有效可行. 而对于制备晶片键合(wafer bonding)的 $\mathrm{LiNbO}_{3}$ 晶体薄膜(厚度通常小于 $\left.1 \mu \mathrm{m}\right)$, 通常采用能量 为几百 $\mathrm{keV}$ 的氦离子注人.

原子序数 $A 8$ 且离子质量比轻离子重的离子一般被 称为重离子, 它们也能够用于在玻璃和晶体等介电材 料中制备高质量的光波导结构 ${ }^{[3,10]}$. 这些离子有时也被 称为“中等质量”离子(典型的离子包括氧、硅等，传统 上把碳、氮等比氢、氦离子重, 但原子序数小于 8 的离 子也归为此类)，以避免与“真实”的重离子(例如 $\mathrm{Au}$ 、 
$\mathrm{Ag}$ 或Xe)混淆. 重离子注人制备光波导结构一般所需的 辐照能量为几到十几 $\mathrm{MeV}$, 离子剂量一般为 $10^{14} \sim 10^{15} \mathrm{~cm}^{-2}$, 比轻离子注人光波导低 $1 \sim 2$ 个数量级 ${ }^{[3]}$. 这是由于单个重离子形成的损伤比单个轻离子大，而 且重离子注人时电子能量损伤 $\left(S_{\mathrm{e}}\right)$ 较大, 其形成折射率 改变的机制是由电子能量损失和核能量损失共同决定 的 ${ }^{[3,10]}$. 重离子辐照也可以用于金属纳米颗粒的合成, 一般需要在介电晶体材料中注人能量为 $150 \sim 500 \mathrm{keV}$ 的金属离子(例如 $\mathrm{Au} 、 \mathrm{Ag} 、 \mathrm{Zn}$ 或 $\mathrm{Cu}$ 等), 典型的辐照离 子剂量为 $10^{16} \sim 10^{17} \mathrm{~cm}^{-2}$. 当注人的金属离子浓度超过 介电材料对其的溶解度时，金属离子会发生聚集形成 金属纳米颗粒结构，在集成光子学、表面增强拉曼光 谱学等领域中具有广泛的应用 ${ }^{[72 ~ 74]}$.

快重离子辐照一般是指能量不小于 $1 \mathrm{MeV} / \mathrm{amu}$ 的 大质量离子(已经使用的离子种类有氯、氩、氪等), 比 重离子注人使用离子的原子量更大 ${ }^{[3]}$. 这里, “快”的概 念是辐照离子能量高, 一般大于几十 $\mathrm{MeV}$ 甚至达到 $\mathrm{GeV}$ 数量级. 因此, 利用快重离子辐照制备光波导的过 程中, 快速重离子辐照的速度效应非常明显, 单个离子 就可以造成非晶化的径迹, 以及足以产生折射率变化 的高强度晶格损伤 ${ }^{[3,10]}$. 相应地，与前文讨论的其他离 子注人相比, 快重离子辐照在制备介电晶体光波导方 面所需要的注人离子剂量很低 $\left(10^{11} \sim 10^{12} \mathrm{ions} / \mathrm{cm}^{2}\right.$ 数量 级), 具有一定的优势 ${ }^{[3,10]}$.

\section{2 离子辐照技术制备的晶体光波导}

载能离子束技术制备的晶体光波导通常可以分为 3类：“光位垒”型、“折射率增强势阱”型和“光位垒+增 强势阱”型(图4) ${ }^{[3,10]}$. 其中, 光位垒型的光波导是最常 见的轻离子注人制备的光波导类型. 由于离子注人诱 导的核能量损伤和晶格损伤主要分布在注人离子的射
程末端，因此非晶化和晶格损伤效应多发生在这个区 域, 伴随产生的是一个折射率降低的薄“光位垒”层, 起 到限制光场传输的作用. “折射率增强势阱”型折射率 分布一般是重离子注人或者快重离子辐照光波导的特 性 ${ }^{[3,10]}$. 与“光位垒”型折射率分布不同, “折射率增强势 阱”型折射率分布是在晶体的浅表层形成厚度较大的 折射率升高区域(由重离子诱导的电子能量损失诱导 形成), 即为波导区域的核心区域. 比较特别的折射率分 布是“光位垒+增强势阱”型，一般出现在轻离子注人和 重离子注人某些双折射晶体的特定偏振方向，例如 $\mathrm{LiNbO}_{3} 、 \beta$-BBO和 $\mathrm{Nd}: \mathrm{YVO}_{4}$ 等 ${ }^{[3,10]}$.

载能离子束辐照技术可以应用于一些电光晶体(例 如 $\mathrm{LiNbO}_{3} 、 \beta-\mathrm{BBO}\left(\beta-\mathrm{Ba}\left(\mathrm{BO}_{2}\right)_{2}\right) 、 \operatorname{KLTN}\left(\mathrm{K}_{1-x} \mathrm{Li}_{x} \mathrm{Ta}_{1-y} \mathrm{Nb}_{y} \mathrm{O}_{3}\right)$ 等)中, 制备并实现基于波导的电光调制器件. 然而, 较 高能量的离子束会影响波导区域中原有的电光性能 (在大多数情况下, 由于离子束引起的晶格损伤和缺陷, 会明显降低波导区域的电光性能). 这一问题通过热退 火等后处理过程可以得到大大缓解，波导区域的晶格 损伤和缺陷会有一定程度的恢复和消除, 这也使得包 括电光性能在内的体材料原有光学性能在波导区域得 到很大程度的恢复(可达体材料电光性能的 $80 \%$ $90 \%$ ). 基于典型的MZI结构, 研究人员已经在载能离子辐照 $\mathrm{LiNbO}_{3}{ }^{[75 \sim 77]}$ 和 $\beta-\mathrm{BBO}^{[78,79]}$ 的光波导结构中制造了电光 调制器件. 其中, 基于 $\mathrm{LiNbO}_{3}$ 光波导的电光调制器在近 红外和可见光波段下都显示了优异的性能，可以达到 体材料中电光系数 $\left(r_{33}\right)$ 的 $90 \%$. 而 $\beta$-BBO波导电光调制 器则主要是在蓝光和紫外光波段运行, 分别得到了最 小值为 $43 \mathrm{~V} \mathrm{~cm}$ (波长为 $257 \mathrm{~nm}$ ) 以及 $79 \mathrm{~V} \mathrm{~cm}$ (波长为 $430 \mathrm{~nm})$ 的半波电压长度积 $\left(V_{\pi} L\right)$.

载能离子束辐照技术也在多种非线性光学晶体光 波导的制备中得到了广泛的应用，最典型的代表性晶
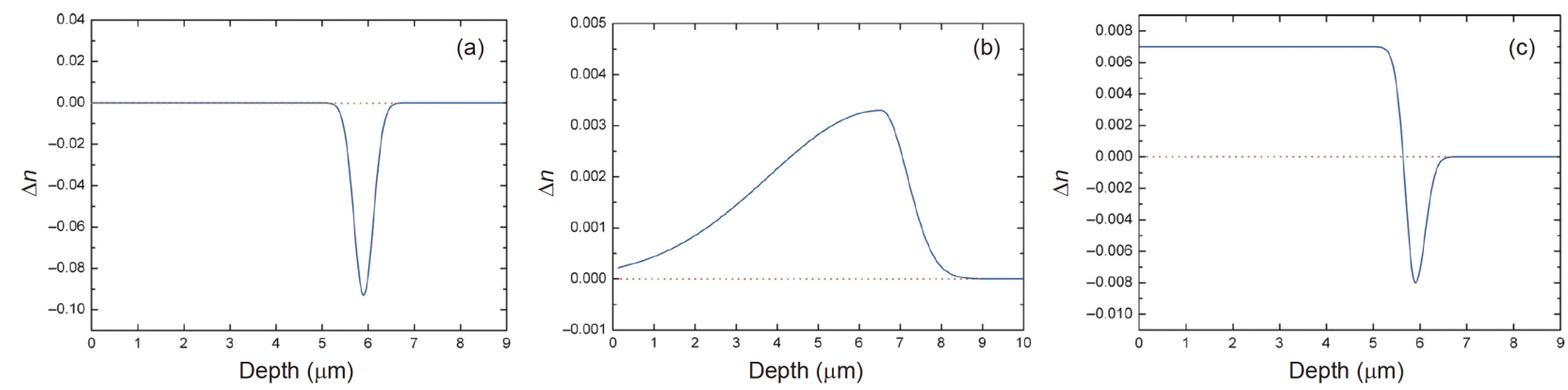

图 4 (网络版彩色)载能离子束技术制备的晶体光波导折射率分布类型 ${ }^{[3]}$. (a) “光位垒”型; (b) “折射率增强势阱”型; (c) “光位垒+增强势阱”型 Figure 4 (Color online) Schematics of the refractive index profiles of ion-beam-processed waveguides ${ }^{[3]}$. (a) "Barrier" type; (b) "buried enhanced well" type; (c) "enhanced well + barrier" type 
体包括 $\mathrm{LiNbO}_{3} 、 \mathrm{KNbO}_{3} 、 \mathrm{KTP} 、 \beta-\mathrm{BBO} 、 \mathrm{LBO}\left(\mathrm{LiB}_{3}\right.$

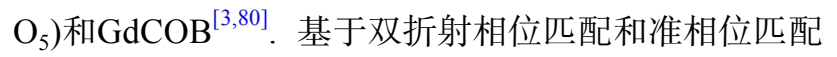
机制, 非线性光学频率转换(主要是倍频效应)也在一 系列离子束加工的晶体光波导中得以实现, 其中最具 代表性的是 $\mathrm{LiNbO}_{3}$ 光波导. 在氢离子注人 $\mathrm{LiNbO}_{3}$ 光波 导中, 二阶非线性系数 $d_{33}$ 与体材料几乎相当; 对于氦 离子注人 $\mathrm{LiNbO}_{3}$ 光波导, $d_{33}$ 则降低至体材料的 $50 \%$ $\sim 80 \%$; 而对于快重离子辐照(例如 $20 \mathrm{MeV} \mathrm{F}, 46 \mathrm{MeV}$ $\mathrm{Cl}, 809 \mathrm{MeV} \mathrm{Kr}$ 或1.4 GeV Xe) $\mathrm{LiNbO}_{3}$ 波导, 其 $d_{33}$ 则降 低至 $60 \% \sim 90 \%{ }^{[3,80]}$. 由于存在大量的晶格损伤和缺陷, 波导势垒区的二阶非线性系数通常有着显著的降 低 ${ }^{[3,80]}$. 对于基于双折射相位匹配的 $\mathrm{MgO}: \mathrm{LiNbO}_{3}$ 波导 (由氦离子注人制备), 其倍频(输出波长为 $567 \mathrm{~nm}$ )的转 换效率为 $0.54 \% \mathrm{~W}^{-1}$. 而在基于准相位匹配的氦离子注 人的周期极化铌酸锂波导中, 转换效率为 $0.01 \% \mathrm{~W}^{-1}$ 的 倍频蓝光(490.5 nm) 和绿光(518.5 nm)已经得到实现, 展现了优于体材料的非线性光学频率转换性能. 除了 $\mathrm{LiNbO}_{3}$ 波导, 离子注入 $\mathrm{KNbO}_{3}$ 波导 ${ }^{[81]}$ 能够实现蓝光输 出的倍频效应, 其最大转换效率为 $12.1 \% \mathrm{~W}^{-1}$, 最大倍 频输出功率为 $320 \mathrm{~mW}$. 此外, 研究人员在氦离子注人 $\mathrm{GdCOB}$ 波导中也得到了转换效率为 $0.4 \% \mathrm{~W}^{-1}$ 的倍频 蓝光输出 (波长为 $411 \mathrm{~nm})^{[82]}$. Ren等人 ${ }^{[83]}$ 报道了快速 重离子辐照的 $\mathrm{Nd}: \mathrm{GdCOB}$ 平面波导, 得到了波长为 $532 \mathrm{~nm}$ 的倍频绿光输出. 在波长为 $1064 \mathrm{~nm}$ 的连续波 和脉冲激光激励下, 倍频光的最大输出功率分别为 $0.53 \mathrm{~mW}$ (在 $334.6 \mathrm{~mW}$ 的连续光洜浦下) 和 $0.72 \mathrm{~mW}$ (在 $102.7 \mathrm{~mW}$ 的脉冲光泵浦下), 转换效率为 $0.48 \% \mathrm{~W}^{-1}$ 和 $6.8 \% \mathrm{~W}^{-1}$.

波导激光也是载能离子束辐照晶体光波导的一 个重要应用, 其基质材料主要是掺杂有活性离子(例 如稀土离子钕、镱、锃等以及金属离子铬、钛等)的 激光晶体. 迄今为止, 载能离子束辐照技术已经在多 种激光晶体材料中制备了波导激光器件 ${ }^{[3,5]}$. 这里, 以 最常见的载能离子束辐照 $\mathrm{Nd}: \mathrm{YAG}$ 波导激光为代表作 简单介绍. 首个离子注人 Nd: YAG波导激光的研究是 利用氦离子注人实现的, 其激光阈值为 $1.6 \mathrm{~mW}$, 斜效 率为 $29 \%$, 最大输出功率为 $4.6 \mathrm{~mW}^{[84]}$. 随后, 研究人 员在优化Nd:YAG波导激光性能方面做出了一系列的 努力, 比较有代表性的是山东大学陈峰课题组 ${ }^{[84 ~ 87]}$ 利 用多种载能离子束技术制备(包括快重离子辐照、质 子写人等)的导波性能和激光性能优异的多类型 $\mathrm{Nd}$ : YAG平面和通道型光波导. 该课题组还创新性地结合
载能离子束技术和飞秒激光烧蚀技术制备了脊型光 波导结构(图 5), 并实现了优于平面光波导的激光性 能 ${ }^{[88]}$.

\section{3 铌酸锂单晶薄膜及波导技术}

铌酸锂单晶薄膜(1ithium-niobate-on-insulator, LNOI)是当前集成光子学中的研究热点之一 ${ }^{[10,12,89 \sim 92]}$. 而基于载能离子束辐照的离子切割技术(ion-cut)则是 铌酸锂单晶薄膜制备过程中的核心环节. 本节将对这 一技术进行简单介绍.

LNOI通常由 $\mathrm{LiNbO}_{3}$ 薄膜层、与 $\mathrm{LiNbO}_{3}$ 薄膜层键 合以提供较大折射率差的低折射率包覆层(通常是绝 缘体层) 以及支撑整个薄膜的衬底层所组成 ${ }^{[10,12]}$. 由于 LNOI薄膜具有大尺寸、高质量和高折射率对比度, 能 够将基于 $\mathrm{LiNbO}_{3}$ 的光子器件/电路的尺寸缩小到亚 $\mu \mathrm{m}$ 级, 极大地推进了 $\mathrm{LiNbO}_{3}$ 在集成光子学中的应用. LNOI技术主要包括三项核心工艺：离子注人、晶片键 合和化学机械抛光 ${ }^{[10,12]}$. 为了在单个芯片上实现更多 光学组件的集成, 厚度均匀的大尺寸LNOI晶片是必要 的. 到目前为止, 直径达4英寸的LNOI晶片已得到商业 化应用. 其中一些晶片参数, 例如表面粗糙度、厚度均 匀性以及 $\mathrm{SiO}_{2}-\mathrm{LN}$ 键合界面的平坦度, 对于高质量晶圆 制造也至关重要. 在LNOI薄膜的制备过程中, 为了得 到能够应用于集成光子学的高质量单晶薄膜, 消除离 子注人引起的缺陷和损伤是非常必要的. LNOI薄膜的 详细制造工艺首先由Günter课题组 ${ }^{[12,93]}$ 提出, 这里将对 其生产流程进行简要介绍. 图6为典型的LNOI晶片制 备流程. 首先, 在 $\mathrm{LiNbO}_{3}$ 晶圆(供体晶圆)上注人能量为 200 400 keV的氦离子, 在离子注人射程的末端形成埋 层的损伤层. 其次, 将厚度为几微米的 $\mathrm{SiO}_{2}$ 层沉积到另 一个 $\mathrm{LiNbO}_{3}$ 晶圆(受体晶圆)上. 第三, 供体和受体晶片 在室温下进行晶片键合. 第四, 在一定的温度(约150 250 C)下进行热退火处理, 从供体晶片上剥离出一层薄薄 的 $\mathrm{LiNbO}_{3}$ 并键合到受体晶片上. 最后, 通过仔细地对 $\mathrm{LiNbO}_{3}$ 薄膜表面进行精密抛光处理, 制备厚度为 300 900 nm的LNOI晶片. 此外, 高质量的LNOI晶片还 需要高温热退火处理(通常在 $\left.400 \sim 500^{\circ} \mathrm{C}\right)$, 在最终的精 细抛光处理之前进一步改善所制备的 $\mathrm{LiNbO}_{3}$ 薄膜质 量. $\mathrm{LiNbO}_{3}$ 薄膜的最终厚度取决于注人的氦离子的能 量和表面抛光处理.

LNOI薄膜能够提供较高的折射率对比度和较强 的光学限制能力, 是制备一系列片上光波导和光子学 

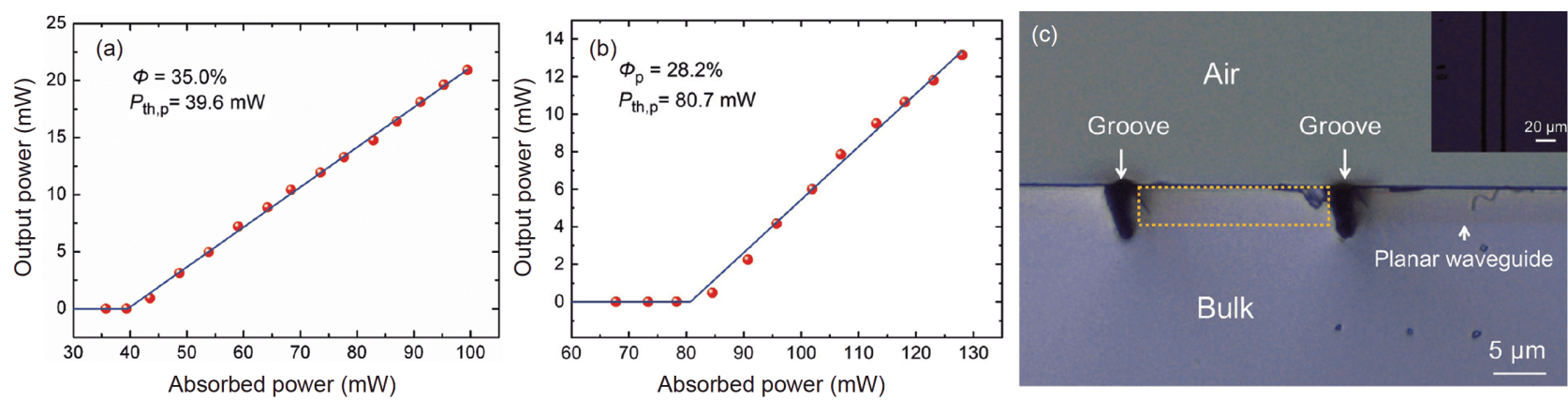

图 5 (网络版彩色)快重离子辐照制备 Nd:YAG平面和脊型波导 ${ }^{[88]} . \mathrm{Nd}: \mathrm{YAG}$ 平面(a)和脊型(b)波导激光(波长 $\left.1.06 \mu \mathrm{m}\right)$ 输出功率随吸收泵浦光(波 长808 nm)功率; (c) 脊型光波导的端面显微镜照片

Figure 5 (Color online) Nd:YAG planar and ridge waveguides fabricated by swift heavy ion irradiation ${ }^{[88]}$. Output laser power at $1.06 \mu \mathrm{m}$ as a function of absorbed pump power at $808 \mathrm{~nm}$ obtained from the Nd:YAG planar (a) and ridge (b) waveguide. (c) Optical microscope image of the end face of the fabricated ridge waveguide

(a)

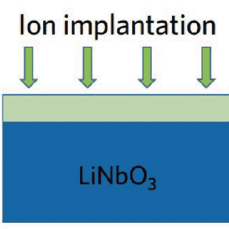

供体

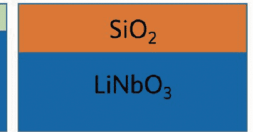

受体 (b)

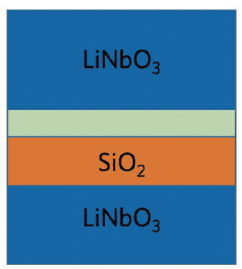

键合

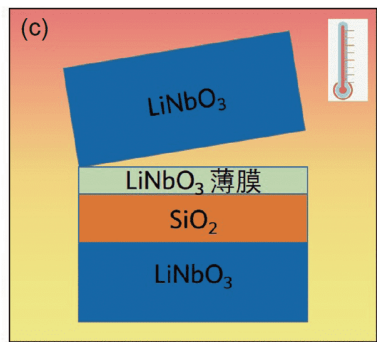

热处理

图 6 (网络版彩色)铌酸锂单晶薄膜的制备流程 ${ }^{[10]}$. (a) 离子注入制备的供体晶片和 $\mathrm{SiO}_{2}$ 薄膜沉积制备的受体晶片示意图; (b) 晶片键合; (c) 高 温热处理

Figure 6 (Color online) Fabrication sequence of LNOI thin film ${ }^{[10]}$. (a) Preparation of ion-implanted donor wafer and $\mathrm{SiO}_{2}$-deposited acceptor wafer; (b) wafer bonding; (c) thermal treatment

器件的理想平台. 随着先进的表面微纳加工技术的迅 猛发展，基于LNOI薄膜平台的多功能片上光子学器件 研究在近年来取得了一系列的成果，基于 $\mathrm{LiNbO}_{3}$ 的高 密度集成光子学芯片也有望在不远的未来得以实 现 $^{[10,12,89-92]}$. 为此, 低损耗的片上光波导的制备尤为重 要, 是构建高性能光子芯片的第一步.

质子交换作为一种用于 $\mathrm{LiNbO}_{3}$ 波导制备的常规且 成熟的技术(通常基于 $\mathrm{LiNbO}_{3}$ 体材料), 也可以直接用于 制造LNOI片上光波导. 尽管质子交换波导的折射率变 化相对较小 (在平面内通常 $<0.1$ ), 但其具有光滑的表面 和边界, 降低了波导的传输损耗(传输损耗低至 $0.2 \mathrm{~dB} / \mathrm{cm})^{[94-96]}$. 值得一提的是，质子交换片上波导的 导波特性通常表现出较强的偏振依赖性，限制了其在 非线性光学中的应用.

与质子交换的片上嵌人式波导不同，干法蚀刻 (dry-etching)制备的片上脊形波导两侧的多余LiNb$\mathrm{O}_{3}$ 已被完全去除, 从而提供了很高的折射率对比度
(在平面内可以 $>1$ ), 可以实现很高的集成度以及紧密 的波导弯曲 ${ }^{[10]}$. 简而言之, 所需的表面波导几何图样 首先通过光刻技术进行定义, 然后通过反应离子束刻 蚀技术将几何图样转移到 $\mathrm{LiNbO}_{3}$ 薄膜层上. 在初步 的制备步骤之后, 为了去除残留的掩模并得到平滑的 波导表面和侧壁,一般需要化学刻蚀方法以及后续的 精细抛光处理. 其中, 最终的精细抛光处理对于获得 低损耗的片上光波导非常重要 ${ }^{[97]}$. 在早期的研究中, 研究人员采用未优化的干法刻蚀和抛光处理制备的 片上LNOI波导的最低传输损耗高达 $10 \mathrm{~dB} / \mathrm{cm}^{[98]}$. 通 过优化干法刻蚀工艺, 该损耗可以降低至 $3 \mathrm{~dB} / \mathrm{cm}^{[99]}$. 值得一提的是, 通过对光刻胶进行多次曝光(由 Lončar课题组 ${ }^{[100]}$ 提出) 并进一步优化干法刻蚀工艺, 具 有 $2.7 \mathrm{~dB} / \mathrm{m}$ 极低传输损耗的LNOI片上波导在近期得 以实现. 在高质量LNOI片上光波导研究的基础上, 针 对LNOI片上回音壁模式光学微腔的研究也逐步展 开. 受制于未优化的制备参数以及精密抛光工艺, 在 
早期研究中，利用干法刻蚀方法制备的LNOI片上光 学微腔在通讯波段的品质因子(quality factor, $Q$ )仅为 $4 \times 10^{3[93,101]}$. 在随后的研究中, 利用优化的干法刻蚀 或飞秒激光烧蚀技术, 片上光学微腔的品质因子得以 突破 $10^{5[102,103]}$. 而这两种制备方法也逐渐发展为当前 高质量LNOI片上光学微腔研究的主要应用技术工 艺. 结合精密的抛光技术, 品质因子高于 $10^{6} \sim 10^{7}$ 的 LNOI片上光学微腔也在近期得到实现 ${ }^{[97,100,104 ~ 106]}$. 其中, 华东师范大学程亚课题组 ${ }^{[106 ~ 108]}$ 开发了飞秒激 光直写与化学机械抛光相结合的光学微腔制备技术, 实现了 $Q$ 值高达 $10^{7}$ 的片上光学微腔以及传输损耗为 $0.027 \mathrm{~dB} / \mathrm{cm}$ 片上波导. 迄今为止, 这些高质量的 LNOI片上波导和光学微腔器件在非线性频率转换、 光频梳产生、超连续、量子芯片等方面都具有重要 的应用并取得了诸多令人瞩目的研究进展 ${ }^{[10,12,89 \sim 92]}$. 例如, Lončar课题组 ${ }^{[109]}$ 成功研制出调制速率高达 210 $\mathrm{Gbit} / \mathrm{s}$ 的片上LNOI电光调制器, 并在CMOS兼容的低 驱动电压 $(1.4 \mathrm{~V})$ 下实现了 $30 \mathrm{GHz} / \mathrm{V}$ 的超宽带宽性能, 打破了传统铌酸锂电光调制器的性能需要在电压和 带宽之间做出折衷的性能限制。该团队还先后在 LNOI片上微环谐振腔中成功实现了覆盖宽度为 $700 \mathrm{~nm}$ 的 $\chi^{(3)}$ 克尔光频梳 ${ }^{[110]}$ 以及可以覆盖整个L波段 (带宽90 nm) 的 $\chi^{(2)}$ 电光光频梳 ${ }^{[111]}$. 此外, Huang课题 组 ${ }^{[112]}$ 和Tang课题组 ${ }^{[113]}$ 先后在片上PPLN光学微腔中 实现了转换效率高达 $230000 \% \mathrm{~W}^{-1}$ 和 $250000 \% \mathrm{~W}^{-1}$ 的 非线性光学倍频过程.

\section{3 总结与展望}

飞秒激光微加工和载能离子束辐照技术在当今微 纳光子学器件和集成光子学的研究中发挥着重要的作 用. 作为两种不同类型的材料改性技术, 它们在基于介 电晶体光波导的研制当中都有着各自独特的优势和特 点, 在光波导结构的集成度、几何形状的灵活度、波 导器件的功能性等方面为多功能集成光子芯片的研究 提供了新的思路. 本文简明扼要地介绍了飞秒激光直 写和载能离子束辐照技术对介电晶体材料改性的物理 机制，并分析了不同类型光波导结构形成的主要机理 以及其导波特性. 在此基础上, 综述了介电晶体光波导 在紧凑型激光光源、非线性光学频率转换器、电光调 制器、集成光子芯片等方面的应用. 在目前已经取得 一系列研究成果的基础上, 我们有理由认为, 通过合理 地优化飞秒激光微加工和载能离子束辐照条件, 以高 性能介电晶体光波导为重要集成元件的多功能光子芯 片和波导网络会对未来的集成光子学和微纳光子学等 多个研究领域产生重要的影响. 今后的研究工作可以 结合不同的微纳加工技术制备以多类型波导结构为基 础的混合型光子学器件展开. 此外, 尽管近年来基于 LNOI薄膜的片上光子学器件发展迅猛, 但距离实现 LNOI多功能集成光子学系统还需要进一步的研究. 未 来的研究方向主要包括低耦合损耗的LNOI片上光学 耦合器、在多波段适用的高转换效率非线性光学频率 转换器、片上偏振调制元件、片上小型光源以及可集 成的光电探测器等.

\section{参考文献}

1 Grivas C. Optically pumped planar waveguide lasers, Part I: Fundamentals and fabrication techniques. Prog Quantum Electron, 2011, 35: 159239

2 Grivas C. Optically pumped planar waveguide lasers: Part II: Gain media, laser systems, and applications. Prog Quantum Electron, 2016, 45-46: $3-160$

3 Chen F. Micro- and submicrometric waveguiding structures in optical crystals produced by ion beams for photonic applications. Laser Photonics Rev, 2012, 6: 622-640

4 Chen F, de Aldana J R V. Optical waveguides in crystalline dielectric materials produced by femtosecond-laser micromachining. Laser Photonics Rev, 2016, 8: 251-275

5 Jia Y C, Chen F. Compact solid-state waveguide lasers operating in the pulsed regime: A review. Chin Opt Lett, 2019, 17: 012302

6 Jia Y, Wang S, Chen F. Femtosecond laser direct writing of flexibly configured waveguide geometries in optical crystals: Fabrication and application. Opto-Electron Adv, 2020, 3: 190042

7 Nikogosyan D N. Nonlinear Optical Crystals: A Complete Survey. New York: Springer, 2005

8 Ferraro P, Grilli S, De Natale P. Ferroelectric Crystals for Photonic Applications. Berlin, Heidelberg: Springer, 2009

9 Kaminskii A A. Laser Crystals: Their Physics and Properties. Berlin, Heidelberg: Springer, 1990

10 Chen F, Amekura H, Jia Y. Ion Irradiation of Dielectrics for Photonic Applications. Singapore: Springer, 2020 
11 Wesch W, Wendler E. Ion Beam Modification of Solids: Ion-Solid Interaction and Radiation Damage. Switzerland: Springer, 2016

12 Poberaj G, Hu H, Sohler W, et al. Lithium niobate on insulator (LNOI) for micro-photonic devices. Laser Photonics Rev, 2012, 6: 488-503

13 Osellame R, Cerullo G, Ramponi R. Femtosecond-laser Micromachining: Photonic and Microfluidic Devices in Transparent Materials. Berlin, Heidelberg: Springer, 2012

14 Sugioka K, Cheng Y. Femtosecond Laser 3D Micromachining for Microfluidic and Optofluidic Applications. London: Springer, 2014

15 Choudhury D, Macdonald J R, Kar A K. Ultrafast laser inscription: Perspectives on future integrated applications. Laser Photonics Rev, 2014, 8: 827-846

16 Gross S, Withford M J. Ultrafast-laser-inscribed 3D integrated photonics: Challenges and emerging applications. Nanophotonics, 2015, 4: 332352

17 Ródenas A, Gu M, Corrielli G, et al. Three-dimensional femtosecond laser nanolithography of crystals. Nat Photonics, 2019, 13: 105-109

18 Zhang B, Wang L, Chen F. Recent advances in femtosecond laser processing of $\mathrm{LiNbO}_{3}$ crystals for photonic applications. Laser Photonics Rev, 2020, 14: 1900407

19 Flamini F, Magrini L, Rab A S, et al. Thermally reconfigurable quantum photonic circuits at telecom wavelength by femtosecond laser micromachining. Light Sci Appl, 2015, 4: e354

20 El Hassan A, Kunst F K, Moritz A, et al. Corner states of light in photonic waveguides. Nat Photonics, 2019, 13: 697-700

21 Ródenas A, Torchia G A, Lifante G, et al. Refractive index change mechanisms in femtosecond laser written ceramic Nd:YAG waveguides: Micro-spectroscopy experiments and beam propagation calculations. Appl Phys B, 2009, 95: 85-96

22 Salamu G, Jipa F, Zamfirescu M, et al. Cladding waveguides realized in Nd:YAG ceramic by direct femtosecond-laser writing with a helical movement technique. Opt Mater Express, 2014, 4: 790-797

23 Thomas J, Heinrich M, Zeil P, et al. Laser direct writing: Enabling monolithic and hybrid integrated solutions on the lithium niobate platform. Phys Status Solidi A, 2011, 208: 276-283

24 Ringleb S, Rademaker K, Nolte S, et al. Monolithically integrated optical frequency converter and amplitude modulator in $\mathrm{LiNbO}_{3}$ fabricated by femtosecond laser pulses. Appl Phys B, 2011, 102: 59-63

25 Liao Y, Xu J, Cheng Y, et al. Electro-optic integration of embedded electrodes and waveguides in $\mathrm{LiNbO}_{3}$ using a femtosecond laser. Opt Lett, 2008, 33: 2281-2283

26 Horn W, Kroesen S, Herrmann J, et al. Electro-optical tunable waveguide Bragg gratings in lithium niobate induced by femtosecond laser writing. Opt Express, 2012, 20: 26922-26928

27 Kroesen S, Horn W, Imbrock J, et al. Electro-optical tunable waveguide embedded multiscan Bragg gratings in lithium niobate by direct femtosecond laser writing. Opt Express, 2014, 22: 23339-23348

28 Burghoff J, Grebing C, Nolte S, et al. Efficient frequency doubling in femtosecond laser-written waveguides in lithium niobate. Appl Phys Lett, 2006, 89: 081108

29 Osellame R, Lobino M, Chiodo N, et al. Femtosecond laser writing of waveguides in periodically poled lithium niobate preserving the nonlinear coefficient. Appl Phys Lett, 2007, 90: 241107

30 Zhang S, Yao J, Shi Q, et al. Fabrication and characterization of periodically poled lithium niobate waveguide using femtosecond laser pulses. Appl Phys Lett, 2008, 92: 231106

31 Thomas J, Heinrich M, Burghoff J, et al. Femtosecond laser-written quasi-phase-matched waveguides in lithium niobate. Appl Phys Lett, 2007, 91: 151108

32 Huang Z, Tu C, Zhang S, et al. Femtosecond second-harmonic generation in periodically poled lithium niobate waveguides written by femtosecond laser pulses. Opt Lett, 2010, 35: 877-879

33 Tan Y, Vázquez de Aldana J R, Chen F. Femtosecond laser-written lithium niobate waveguide laser operating at 1085 nm. Opt Eng, 2014, 53: 107109

34 Lee Y L, Yu N E, Jung C, et al. Second-harmonic generation in periodically poled lithium niobate waveguides fabricated by femtosecond laser pulses. Appl Phys Lett, 2006, 89: 171103

35 Wang Y, Petrov V, Ding Y J, et al. Ultrafast generation of blue light by efficient second-harmonic generation in periodically-poled bulk and waveguide potassium titanyl phosphate. Appl Phys Lett, 1998, 73: 873-875

36 Laurell F, Calmano T, Müller S, et al. Laser-written waveguides in KTP for broadband Type II second harmonic generation. Opt Express, 2012, 20: 22308-22313

37 Campbell S, Thomson R R, Hand D P, et al. Frequency-doubling in femtosecond laser inscribed periodically-poled potassium titanyl phosphate waveguides. Opt Express, 2007, 15: 17146-17150

38 Zhang S, Yao J, Liu W, et al. Second harmonic generation of periodically poled potassium titanyl phosphate waveguide using femtosecond laser pulses. Opt Express, 2008, 16: 14180-14185 
39 Beecher S J, Thomson R R, Reid D T, et al. Strain field manipulation in ultrafast laser inscribed $\mathrm{BiB}_{3} \mathrm{O}_{6}$ optical waveguides for nonlinear applications. Opt Lett, 2011, 36: 4548-4550

40 Jia Y, Aldana J R V, Romero C, et al. Femtosecond-laser-inscribed $\mathrm{BiB}_{3} \mathrm{O}_{6}$ nonlinear cladding waveguide for second-harmonic generation. Appl Phys Express, 2012, 5: 072701

41 Jia Y, Vázquez de Aldana $\mathrm{J}$ R, Lu Q, et al. Second harmonic generation of violet light in femtosecond-laser-inscribed $\mathrm{BiB}_{3} \mathrm{O}_{6}$ cladding waveguides. Opt Mater Express, 2013, 3: 1279-1284

42 Jia Y, Chen F, Vázquez de Aldana J R, et al. Femtosecond laser micromachining of Nd:GdCOB ridge waveguides for second harmonic generation. Opt Mater, 2012, 34: 1913-1916

43 Wang L, Zhang X, Li L, et al. Second harmonic generation of femtosecond laser written depressed cladding waveguides in periodically poled $\mathrm{MgO}: \mathrm{LiTaO}_{3}$ crystal. Opt Express, 2019, 27: 2101-2111

44 Li L, Romero C, Vázquez de Aldana J R, et al. Efficient quasi-phase-matching in fan-out PPSLT crystal waveguides by femtosecond laser direct writing. Opt Express, 2019, 27: 36875-36885

45 Zhang B, Li L, Wang L, et al. Second harmonic generation in femtosecond laser written lithium niobate waveguides based on birefringent phase matching. Opt Mater, 2020, 107: 110075

46 Nie W, Jia Y, Vázquez de Aldana J R, et al. Efficient second harmonic generation in 3D nonlinear optical-lattice-like cladding waveguide splitters by femtosecond laser inscription. Sci Rep, 2016, 6: 22310

47 Dong N, Martínez de Mendivil J, Cantelar E, et al. Self-frequency-doubling of ultrafast laser inscribed neodymium doped yttrium aluminum borate waveguides. Appl Phys Lett, 2011, 98: 181103

48 Ren Y, Chen F, Vázquez de Aldana J R. Near-infrared lasers and self-frequency-doubling in Nd:YCOB cladding waveguides. Opt Express, 2013, 21: $11562-11567$

49 Calmano T, Siebenmorgen J, Reichert F, et al. Crystalline $\operatorname{Pr}_{\mathrm{SrAl}} \mathrm{Sr}_{12} \mathrm{O}_{19}$ waveguide laser in the visible spectral region. Opt Lett, 2011, 36: 46204622

50 Müller S, Calmano T, Metz P, et al. Femtosecond-laser-written diode-pumped Pr:LiYF 4 waveguide laser. Opt Lett, 2012, 37: 5223-5225

51 Benayas A, Jaque D, McMillen B, et al. Thermal stability of microstructural and optical modifications induced in Sapphire by ultrafast laser filamentation. J Appl Phys, 2010, 107: 033522

52 Calmano T, Siebenmorgen J, Hellmig O, et al. Nd:YAG waveguide laser with $1.3 \mathrm{~W}$ output power, fabricated by direct femtosecond laser writing. Appl Phys B, 2010, 100: 131-135

53 Tan Y, Rodenas A, Chen F, et al. 70\% slope efficiency from an ultrafast laser-written Nd:GdVO 4 channel waveguide laser. Opt Express, 2010, 18: 24994-24999

54 Okhrimchuk A G, Shestakov A V, Khrushchev I, et al. Depressed cladding, buried waveguide laser formed in a YAG:Nd ${ }^{3+}$ crystal by femtosecond laser writing. Opt Lett, 2005, 30: 2248-2250

55 Liu H, Jia Y, Vázquez de Aldana J R, et al. Femtosecond laser inscribed cladding waveguides in Nd:YAG ceramics: Fabrication, fluorescence imaging and laser performance. Opt Express, 2012, 20: 18620-18629

56 Ren Y, Dong N, MacDonald J, et al. Continuous wave channel waveguide lasers in Nd:LuVO fabricated by direct femtosecond laser writing. Opt Express, 2012, 20: 1969-1974

57 Ren Y, Vázquez de Aldana J R, Chen F, et al. Channel waveguide lasers in Nd:LGS crystals. Opt Express, 2013, 21: 6503-6508

58 Nie W, Cheng C, Jia Y, et al. Dual-wavelength waveguide lasers at 1064 and $1079 \mathrm{~nm}$ in Nd:YAP crystal by direct femtosecond laser writing. Opt Lett, 2015, 40: 2437-2440

59 Jia Y, Cheng C, Vázquez de Aldana J R, et al. Monolithic crystalline cladding microstructures for efficient light guiding and beam manipulation in passive and active regimes. Sci Rep, 2014, 4: 5988

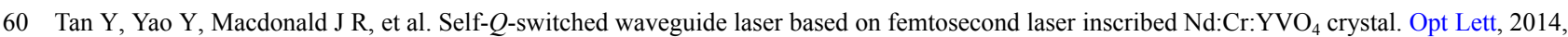
39: 5289-5292

61 Liu H, Cheng C, Romero C, et al. Graphene-based Y-branch laser in femtosecond laser written Nd:YAG waveguides. Opt Express, 2015, 23: 9730-9735

62 Nie W, He R, Cheng C, et al. Optical lattice-like cladding waveguides by direct laser writing: Fabrication, luminescence, and lasing. Opt Lett, 2016, 41: 2169-2172

63 Liu H, Vázquez de Aldana J R, Hong M, et al. Femtosecond laser inscribed Y-branch waveguide in Nd:YAG crystal: Fabrication and continuouswave lasing. IEEE J Sel Top Quantum Electron, 2016, 22: 4500204

64 Jia Y, Cheng C, Vazquez de Aldana J R, et al. Three-dimensional waveguide splitters inscribed in Nd:YAG by femtosecond laser writing: Realization and laser emission. J Lightwave Technol, 2016, 34: 1328-1332

65 Bain F M, Lagatsky A A, Thomson R R, et al. Ultrafast laser inscribed $\mathrm{Yb}: \mathrm{KGd}\left(\mathrm{WO}_{4}\right)_{2}$ and $\mathrm{Yb}: \mathrm{KY}\left(\mathrm{WO}_{4}\right)_{2}$ channel waveguide lasers. Opt 
Express, 2009, 17: 22417-22422

66 Calmano T, Müller S. Crystalline waveguide lasers in the visible and near-infrared spectral range. IEEE J Sel Top Quantum Electron, 2015, 21: 1602213

67 Calmano T, Siebenmorgen J, Paschke A G, et al. Diode pumped high power operation of a femtosecond laser inscribed Yb:YAG waveguide laser. Opt Mater Express, 2011, 1: 428-433

68 Jia Y, Vázquez de Aldana J R, Chen F. Efficient waveguide lasers in femtosecond laser inscribed double-cladding waveguides of Yb:YAG ceramics. Opt Mater Express, 2013, 3: 645-650

69 Ren Y, Brown G, Ródenas A, et al. Mid-infrared waveguide lasers in rare-earth-doped YAG. Opt Lett, 2012, 37: 3339-3341

70 Tan Y, Jia Y, Chen F, et al. Simultaneous dual-wavelength lasers at $1064 \mathrm{~nm}$ and $1342 \mathrm{~nm}$ in femtosecond-laser-written $\mathrm{Nd} \mathrm{YVO}_{4}$ channel waveguides. J Opt Soc Am B, 2011, 28: 1607-1610

71 Ren Y Y, Brown G, Mary R, et al. 7.8-GHz graphene-based 2- $\mu$ m monolithic waveguide laser. IEEE J Sel Top Quantum Electron, 2015, 21: 395400

72 Li R, Pang C, Li Z, et al. Fused silica with embedded 2D-like Ag nanoparticle monolayer: Tunable saturable absorbers by interparticle spacing manipulation. Laser Photonics Rev, 2020, 14: 1900302

73 Li R, Pang C, Li Z, et al. Plasmonic nanoparticles in dielectrics synthesized by ion beams: Optical properties and photonic applications. Adv Opt Mater, 2020, 8: 1902087

74 Pang C, Li R, Li Z, et al. A novel hierarchical nanostructure for enhanced optical nonlinearity based on scattering mechanism. Small, 2020, 16: 2003172

75 Destefanis G L, Gailliard J P, Ligeon E L, et al. The formation of waveguides and modulators in $\mathrm{LiNbO}_{3}$ by ion implantation. J Appl Phys, 1979, 50: 7898-7905

76 Bentini G G, Bianconi M, Cerutti A, et al. Integrated Mach-Zehnder micro-interferometer on $\mathrm{LiNbO}_{3}$. Opt Lasers Eng, 2007, 45: 368-372

77 Bentini G G, Bianconi M, Cerutti A, et al. A new miniaturised optical system for chemical species spectroscopic detection based on a scanning integrated Mach-Zehnder microinterferometer on $\mathrm{LiNbO}_{3}$. Orig Life Evol Biosph, 2006, 36: 597-603

78 Poberaj G, Degl'Innocenti R, Medrano C, et al. UV integrated optics devices based on beta-barium borate. Opt Mater, 2009, 31: 1049-1053

79 Degl'Innocenti R, Majkic A, Vorburger $\mathrm{P}$, et al. Ultraviolet electro-optic amplitude modulation in $\beta$ - $\mathrm{BaB}_{2} \mathrm{O}_{4}$ waveguides. Appl Phys Lett, 2007, 91: 051105

80 Chen F. Photonic guiding structures in lithium niobate crystals produced by energetic ion beams. J Appl Phys, 2009, 106: 081101

81 Fluck D, Gunter P. Second-harmonic generation in potassium niobate waveguides. IEEE J Sel Top Quantum Electron, 2000, 6: 122-131

82 Vincent $\mathrm{B}$, Boudrioua A, Loulergue $\mathrm{J} \mathrm{C}$, et al. Channel waveguides in $\mathrm{Ca}_{4} \mathrm{GdO}\left(\mathrm{BO}_{3}\right)_{3}$ fabricated by $\mathrm{He}^{+}$implantation for blue-light generation. Opt Lett, 2003, 28: 1025-1027

83 Ren Y, Jia Y, Chen F, et al. Second harmonic generation of swift carbon ion irradiated Nd:GdCOB waveguides. Opt Express, 2011, 19: 12490

84 Field S J, Hanna D C, Large A C, et al. Low threshold ion-implanted Nd:YAG channel waveguide laser. Electron Lett, 1991, 27: 2375-2376

85 Ren Y, Dong N, Chen F, et al. Swift heavy-ion irradiated active waveguides in Nd:YAG crystals: Fabrication and laser generation. Opt Lett, 2010, 35: 3276-3278

86 Ren Y, Dong N, Chen F, et al. Swift nitrogen ion irradiated waveguide lasers in Nd:YAG crystal. Opt Express, 2011, 19: 5522-5527

87 Yao Y, Tan Y, Dong N, et al. Continuous wave Nd:YAG channel waveguide laser produced by focused proton beam writing. Opt Express, 2010, 18: 24516-24521

88 Jia Y, Dong N, Chen F, et al. Continuous wave ridge waveguide lasers in femtosecond laser micromachined ion irradiated Nd:YAG single crystals. Opt Mater Express, 2012, 2: 657-662

89 Boes A, Corcoran B, Chang L, et al. Status and potential of lithium niobate on insulator (LNOI) for photonic integrated circuits. Laser Photonics Rev, 2018, 12: 1700256

90 Honardoost A, Abdelsalam K, Fathpour S. Rejuvenating a versatile photonic material: Thin-film lithium niobate. Laser Photonics Rev, 2020, 14: 2000088

91 Qi Y, Li Y. Integrated lithium niobate photonics. Nanophotonics, 2020, 9: 1287-1320

92 Lin J, Bo F, Cheng Y, et al. Advances in on-chip photonic devices based on lithium niobate on insulator. Photonics Res, 2020, 8: 1910-1936

93 Poberaj G, Koechlin M, Sulser F, et al. Ion-sliced lithium niobate thin films for active photonic devices. Opt Mater, 2009, 31: 1054-1058

94 Cai L, Han S L H, Hu H. Waveguides in single-crystal lithium niobate thin film by proton exchange. Opt Express, 2015, 23: 1240-1248

95 Cai L, Wang Y, Hu H. Low-loss waveguides in a single-crystal lithium niobate thin film. Opt Lett, 2015, 40: 3013-3016

96 Cai L, Kong R, Wang Y, et al. Channel waveguides and Y-junctions in $x$-cut single-crystal lithium niobate thin film. Opt Express, 2015, 23: $29211-29221$

97 Wolf R, Breunig I, Zappe H, et al. Scattering-loss reduction of ridge waveguides by sidewall polishing. Opt Express, 2018, 26: 19815-19820 
$98 \mathrm{Hu} \mathrm{H}$, Ricken R, Sohler W. Lithium niobate photonic wires. Opt Express, 2009, 17: 24261-24268

99 Wang C, Xiong X, Andrade N, et al. Second harmonic generation in nano-structured thin-film lithium niobate waveguides. Opt Express, 2017, 25 : 6963-6973

100 Zhang M, Wang C, Cheng R, et al. Monolithic ultra-high- $Q$ lithium niobate microring resonator. Optica, 2017, 4: 1536-1537

101 Guarino A, Poberaj G, Rezzonico D, et al. Electro-optically tunable microring resonators in lithium niobate. Nat Photonics, 2007, 1: 407-410

102 Lin J T, Xu Y X, Fang Z W, et al. Second harmonic generation in a high- $Q$ lithium niobate microresonator fabricated by femtosecond laser micromachining. Sci China-Phys Mech Astron, 2015, 58: 114209

103 Wang C, Burek M J, Lin Z, et al. Integrated high quality factor lithium niobate microdisk resonators. Opt Express, 2014, 22: 30924-30933

104 Desiatov B, Shams-Ansari A, Zhang M, et al. Ultra-low-loss integrated visible photonics using thin-film lithium niobate. Optica, 2019, 6: 380384

105 Wolf R, Breunig I, Zappe H, et al. Cascaded second-order optical nonlinearities in on-chip micro rings. Opt Express, 2017, 25: 29927-29933

$106 \mathrm{Wu}$ R, Zhang J, Yao N, et al. Lithium niobate micro-disk resonators of quality factors above 107. Opt Lett, 2018, 43: 4116-4119

107 Wang M, Wu R, Lin J, et al. Chemo-mechanical polish lithography: A pathway to low loss large-scale photonic integration on lithium niobate on insulator. Quantum Eng, 2019, 1: e9

$108 \mathrm{Wu}$ R, Wang M, Xu J, et al. Long low-loss-litium niobate on insulator waveguides with sub-nanometer surface roughness. Nanomaterials, 2018, 8: 910

109 Wang C, Zhang M, Chen X, et al. Integrated lithium niobate electro-optic modulators operating at CMOS-compatible voltages. Nature, 2018, 562: 101-104

110 Wang C, Zhang M, Yu M, et al. Monolithic lithium niobate photonic circuits for Kerr frequency comb generation and modulation. Nat Commun, 2019, 10: 978

111 Zhang M, Buscaino B, Wang C, et al. Broadband electro-optic frequency comb generation in a lithium niobate microring resonator. Nature, 2019, 568: 373-377

112 Chen J Y, Ma Z H, Sua Y M, et al. Ultra-efficient frequency conversion in quasi-phase-matched lithium niobate microrings. Optica, 2019, 6: $1244-1245$

$113 \mathrm{Lu}$ J, Surya J B, Liu X, et al. Periodically poled thin-film lithium niobate microring resonators with a second-harmonic generation efficiency of 250,000\%/W. Optica, 2019, 6: 1455-1460 


\title{
Fabrication and applications of dielectric optical crystalline waveguides
}

\author{
Yuechen Jia, Shixiang Wang, Yang Tan, Lei Wang \& Feng Chen \\ School of Physics, State Key Laboratory of Crystal Materials, Jinan 250100, China \\ * Corresponding author, E-mail: drfchen@sdu.edu.cn
}

Optical waveguides, being defined as high refractive index cores surrounded by low-index layers, are the basic component for multi-functional integrated photonic systems. Generally, optical waveguide structures could confine the light propagation within very small regions of orders of several micrometers, in which the light intensities could reach very high levels with respect to the bulk materials. Benefiting from this exceptional advantageous, capability of some features (e.g. nonlinear response, lasing performances, etc.) of many materials may be considerably enhanced to a certain extent in waveguide structures, enabling the implementations of highly efficient miniaturized and integrated platforms for a variety of photonic applications. In practice, channel (two dimensional, 2D) waveguides are more attractive than planar (one dimensional, 1D) waveguide structures because the former could restrict the light propagation in two dimensions, reaching higher optical density in more compact geometries, and are easier for constructions of more compact functional integrated devices.

Dielectric crystals play important roles in many fields of optics and photonics. For example, nonlinear crystals are important frequency converters of light at different wavelength regions. Laser crystals are the favorite and attractive gain mediums for solid-state laser systems with comparable low lasing thresholds and excellent thermal properties with respect to glasses. Electro-optic crystals are ideal platforms for the modulations of light phase, energy and polarizations. Up to now, various optical applications have been realized through a broad variety of crystal-based devices and components. Benefiting from this, crystalline waveguides, with the combination of compact geometry of channel waveguides and valuable features of crystals, could become unique platforms for versatile miniature and integrated photonic applications.

As of yet, a few methods have been employed to fabricate channel waveguides in crystals, including femtosecond (fs) laser micromachining, focus proton beam writing, ion implantation/irradiation, etc. Among these, fs laser micromachining has recently emerged as one of the most efficient techniques for direct three-dimensional microfabrication of transparent optical materials. During this process, fs laser pulses focused beneath the surface of a crystal are absorbed through nonlinear photoionization mechanisms, giving rise to a permanent localized modification with dimensions on the order of micro or submicrometre. By a suitable adjustment of the irradiation parameters, one could induce a localized refractive index modification, enabling direct channel waveguide fabrication by simple scanning the bulk materials, showing wide applicability in a large number of materials. Ion implantation/irradiation is also a powerful and efficient method for waveguide construction, which has been proved successful in more than 100 materials. Generally, ion implantation/ irradiation influences the refractive indices of the target materials mainly via physical mechanisms, which are independent on the chemical properties of the target materials. Benefiting this unique feature, ion implantation/irradiation has a wide applicability to most optical materials. Particularly, by combinations of fs laser micromachining or precise diamond dicing, 2D channel waveguide structure could be efficiently fabricated on the surface of the substrate.

In this review, we will introduce femtosecond laser direct writing and energetic ion irradiation of crystalline waveguides (including crystalline thin films) and their related waveguide properties. The applications of dielectric waveguides in laser generation, nonlinear optical frequency conversion, signal modulation, and quantum information are reviewed.

dielectric crytals, optical waveguides, femtosecond laser micromachining, ion implantation, lithium niobate on insulator (LNOI)

doi: 10.1360/TB-2020-1686 